\title{
Effect of autologous hematopoietic stem cell transplant on the development of second primary malignancies in multiple myeloma patients
}

\author{
A. S. Rosenberg $\mathbb{D}^{1,2}$, A. Brunson', J. Tuscano ${ }^{2,3}$, B. A. Jonas $\mathbb{D}^{2,3}$, R. Hoeg ${ }^{2}$, T. Wun $\mathbb{B}^{1,2,3}$ and T. H. M. Keegan ${ }^{1,2}$
}

\begin{abstract}
Autologous stem cell transplant (aHSCT) is associated with improved survival for multiple myeloma (MM) patients but may be associated with second primary malignancy (SPM) development. Using the California Cancer Registry linked to statewide hospitalization data, we determined the cumulative incidence (CMI) of SPMs more than 1 year after MM diagnosis, accounting for the competing risk of death. AHSCT recipients were matched 1:2 to non-aHSCT patients. Adjusted hazard ratios (aHR) were estimated using the Fine and Gray method. Among 16,331 patients, 933 (5.7\%) developed a SPM more than 1 year after diagnosis. The 10-year CMI of developing any SPM was 6.6\%, 5.7\% for solid tumor SPM and $0.9 \%$ for hematologic malignancies. The 10-year CMI of developing any SPM was similar among aHSCT [9.1\% (7.7-10.7\%)] and non-aHSCT [7.5\% (6.5-8.6\%)] $(P=0.26)$ recipients and there was no difference in solid-tumor SPMs $(P=0.98)$. The 10-year CMI of hematologic SPMs was higher among aHSCT recipients $[2.1 \%(1.4-2.9 \%)$ vs. $0.8 \%$ $(0.5-1.2 \%) ; P=0.005]$, corresponding to a $1.3 \%$ absolute increase and an aHR of 1.51 (1.01-2.27). Ten-year myelomaspecific and non-cancer mortality rates were 59\% (58.2-60.0\%) and 18.1\% (17.4-18.8\%), respectively. Although aHSCT was associated with a small increase in hematologic SPMs, mortality was driven by MM and non-cancer causes.
\end{abstract}

\section{Introduction}

Multiple myeloma survival has improved dramatically over the last 20 years due to adoption of autologous stem cell transplant (aHSCT) and multiple new drug classes for treatment ${ }^{1-7}$. In the era of novel agents, randomized phase 3 trials have confirmed survival benefits to aHSCT ${ }^{8-12}$. As the survival of multiple myeloma patients improves, late effects are now being recognized and need to be addressed ${ }^{13,14}$. Multiple myeloma patients have been found to be at an increased risk of second primary malignancies (SPMs) compared to the general population ${ }^{15-27}$, with treatments, notably the immunomodulatory agents and alkylator based

\footnotetext{
Correspondence: A S. Rosenberg (asrosenberg@ucdavis.edu)

${ }^{1}$ Davis School of Medicine, Center for Hematology and Oncology Outcomes Research and Training (COHORT), University of California, Sacramento, CA, USA ${ }^{2}$ Davis Comprehensive Cancer Center, University of California, Sacramento, CA, USA

Full list of author information is available at the end of the article
}

chemotherapy, likely contributing to this increased risk $^{16,20,22,23,28-30}$.

Randomized controlled trials (RCTs) of aHSCT use in the modern treatment era have compared early to delayed aHSCT, but have not compared SPM development in these two $\mathrm{arms}^{8-10}$. Lenalidomide maintenance trials post-aHSCT have found higher SPM rates in the maintenance arms, but cannot address the potential additional risk contributed by high dose chemotherapy ${ }^{19,31-33}$.

Cancer registry-based studies have used diagnosis date as a proxy for exposure to aHSCT with conflicting results $^{23,34}$. Analyses of large stem cell transplant registries comparing SPM incidence among aHSCT recipients to general population cancer incidence rates found the risks of acute lymphoblastic leukemia, acute myeloid leukemia, Hodgkin lymphoma were increased ${ }^{15,20}$. However these studies relied on comparisons of rates in different registries, and cross-registry comparisons may be confounded. No prior population level studies have 
directly compared the incidence of SPMs in myeloma patients, who did and did not undergo an aHSCT.

To address these gaps in the literature, we utilized longitudinal data on multiple myeloma patients from the population-based California Cancer Registry (CCR) linked to California Patient Discharge Database (PDD) and Ambulatory Surgery (AS) database to identify those undergoing aHSCT. Findings from this study will provide valuable insight into the potential late effects of aHSCT to better inform patient decisions.

\section{Methods}

\section{Databases}

This retrospective observational cohort study utilized linked data between the CCR and California PDD and AS databases, which are maintained and previously linked by The Office of Statewide Health Planning and Development. The CCR is a statewide population-based cancer surveillance system collecting cancer incidence and mortality information since 1988; it captures $>98 \%$ of all cancer diagnoses in the state excluding non-invasive squamous and basal cell carcinomas of the skin. From the CCR, we obtained date of diagnosis, initial course of treatment, and patient demographics, including race/ ethnicity, gender, age, location of residence (rural vs. urban), marital status, neighborhood socioeconomic status $^{35}$, and insurance type at time of diagnosis ${ }^{36}$. The PDD captures information on all discharges from non-federal hospitals in California since 1991. Beginning in 2005, the AS database, including all hospital associated AS facilities, has also been mandated. The databases were linked at the patient level using the record linkage number (RLN), an encrypted form of social security number. The RLN allows serial linking of multiple hospitalization records over time. Patients who did not have a RLN (11\%) or were only reported by Department of Veterans Affairs (which does not send data to the PDD or AS, and provides data to CCR but cannot be disclosed for research purposes) were excluded. Both PDD and AS include up to 25 diagnoses and up to 21 procedures associated with each hospitalization, coded using the International Classification of Disease, Ninth Revision, Clinical Modification (ICD-9$\mathrm{CM}$ ) in the PDD and Current Procedural Terminology (CPT) in the AS. Each procedure code has a date, and were used to ascertain aHSCT and comorbidities.

\section{Patients}

Between 1991-2014, patients with first primary multiple myeloma patients were identified in CCR using ICD-O-3 histology code $9732^{37}$. To account for surveillance bias and immortal time bias, all patients had to have at least 1 year of follow-up time recorded, and all patients who developed a SPM during the first year after diagnosis were excluded.

\section{Exposure}

Only first aHSCT use was examined, and was considered present if it was identified in either the CCR, PDD or AS, and was considered "early", if performed within 12 months of diagnosis, or "late", if greater than 12 months, as done previously ${ }^{12}$. Year of diagnosis was categorized into four treatment eras (1991-1997: preimmunomodulatory (imid) and infrequent aHSCT use, 1998-2002: increased imid and aHSCT use, 2003-2007: introduction of proteasome inhibitors (PI) and second generation imids, 2008-2013: modern combination imid and PI more commonly used $)^{34}$. Comorbidities were captured in the PDD up to 2 years prior to the multiple myeloma diagnosis date. They were identified using the Elixhauser index, excluding cancer ${ }^{38}$, and categorized as no admissions in PDD within the 2 prior years (and thus no information), 0 comorbidities, 1-2 comorbidities, and $\geq 3$ comorbidities. Neighborhood socioeconomic status (nSES) is measured at the neighborhood level by the CCR, and divided into quintiles ${ }^{35}$. First-course chemotherapy and radiation are captured as a binary variables without details of regimen or dosing.

\section{Outcome}

First SPM was obtained from the CCR and classified using SEER site recode. Non-invasive squamous and basal cell carcinomas of this skin are not reportable to the CCR and were not included, while in situ breast cancers were included. No additional malignancies beyond the second were considered in this study. First SPM was evaluated as overall, solid, and hematologic. Cause specific mortality was assessed using cause of death ascertained from death certificates by CCR.

\section{Statistics}

The cumulative incidence (CMI) of developing any SPM, solid tumors, and hematologic malignancies, and associated 95\% confidence intervals were computed, accounting for the competing risk of death. The cumulative incidence of multiple myeloma specific mortality, non-cancer mortality, and SPM development were compared using the competing risk framework. To compare the conditional cumulative incidence of SPMs between aHSCT recipients and those without aHSCT, patients were matched 1:2 on sex, age $+/-3$ years, year of diagnosis $+/-2$ years, race/ethnicity, nSES status (quintiles), Elixhauser comorbidity index (NA, 0, 1-2, 3+), and follow-up time (Supplemental Table S1). Multivariable Cox proportional hazards regression models, using the methods of Fine and Gray to account for competing risk of death, estimated the effect of aHSCT on the risk of SPM development accounting for baseline demographics. Autologous HSCT was included as a time dependent covariate. The proportional hazard assumption was 
assessed using Schoenfeld residuals ${ }^{39}$, and models were stratified for variables that did not meet the proportional hazards assumption. All calculations were using SAS (version 9.4, Cary, NC).

This study was reviewed by the Committee of the Protection of Human Subjects, which serves as the Institutional Review Board for the California Health and Human Services Agency, and by the University of California Davis Institutional Review Board.

\section{Results}

\section{Patient characteristics}

Between 1991 and 2013, 16,331 patients with multiple myeloma were identified within the CCR and met the inclusion criteria (Table 1). The median age at diagnosis was 66 years. There was a slight male predominance (54\%). Non-Hispanic whites made up the largest proportion of patients (59\%), followed by Hispanics (18\%), African Americans (13\%), and Asians (9\%). Autologous HSCT was utilized in $19.6 \%$ of newly diagnosed patients, more commonly among men, younger patients, those with fewer comorbidities, higher neighborhood socioeconomic status and private insurance, and less commonly among African Americans and those with Medicare, no or unknown insurance. The use of aHSCT increased over time.

\section{Second primary malignancy (SPM)}

SPMs were identified in 933 (5.7\%) patients (Table 1). The median time from the diagnosis of $\mathrm{MM}$ to the diagnosis of SPMs was 3.8 years (range 1.0-24.5 years). Solid tumors were more commonly diagnosed than hematologic malignancies, with gastrointestinal, breast, and male genitourinary cancers being the most common. Among hematologic malignancies, leukemia was the most common, accounting for $8.7 \%$ of SPMs. Among these, acute myeloid and acute lymphoid leukemia were the most commonly diagnosed accounting for $58 \%$ and $21 \%$ of leukemia diagnoses, respectively. Myelodysplastic syndrome was captured by the CCR starting in 2002, accounting for $3.3 \%$ of all SPMs.

The cumulative incidence of developing any SPM was $4.0 \%(3.7-4.3 \%)$ at 5 years and $6.6 \%(6.2-7.1 \%)$ at 10 years after diagnosis (Fig. 1). Multiple myeloma-related mortality was $41.8 \%(41.0-42.6 \%)$ and $59.1 \%(58.2-60.0 \%)$ and non-cancer related mortality was $11.6 \%(11.0-12.1 \%)$ and $18.1 \%(17.4-18.8 \%)$ at 5 and 10 years, respectively. By comparison, death attributable to SPMs were $1.9 \%$ $(1.7-2.1 \%)$ and $3.0 \%(2.7-3.3 \%)$.

Rates of SPM development changed during the different treatment eras. The 5-year CMI rate for patients diagnosed 2008-2013 was 5.0\% (4.3-5.8\%) compared to $3.8 \%$ $(3.2-4.4 \%), \quad 3.9 \% \quad(3.3-4.6 \%), \quad 3.4 \% \quad(2.9-4.0 \%) \quad$ in 2003-2007, 1998-2002, and 1991-1997, respectively
$(P<0.001)$ (Fig. 2). Median time to develop a SPM was similar in the earlier three eras: 54 months, 52 months, and 53 months for those diagnosed 1991-1997, 1998-2002, and 2003-2007, respectively. In contrast, the median time to SPM in patients diagnosed 2008-2013 was 31 months, consistent with shorter follow-up time in the more recent cohort. Because myelodysplastic syndrome (MDS) was only recorded starting in 2002, we performed a sensitivity analysis excluding MDS and observed similar findings (Supplemental Fig. 1).

\section{Association of second primary malignancy and aHSCT use}

Patients undergoing aHSCT did not have an increased risk of any SPMs, with 5-year and 10-year CMI rates of $4.8 \%(3.9-5.9 \%)$ and $9.1 \%(7.7-10.7 \%)$ compared to $4.7 \%$ $(4.0-5.5 \%)$ and $7.5 \%(6.5-8.6 \%)$, respectively $(P=0.26)$ among non-aHSCT patients (Fig. 3a). In multivariable analysis, this corresponded to an adjusted hazard ratio of 1.13 (0.94-1.4, $P=0.19$ ) (Fig. 4). We then examined the association between aHSCT use and the development of solid tumor SPMs and hematologic SPMs. The 5-year and 10-year CMI of developing any solid tumor SPM among aHSCT recipients was $3.6 \%(2.8-4.5 \%)$ and $6.7 \%$ $(5.5-8.1 \%)$ compared to $3.9 \%(3.3-4.7 \%)$ and $6.5 \%$ (5.5-7.6\%) among non-aHSCT recipients (Fig. 3b) $(P=$ $0.90)$. This corresponded to an aHR of $1.03(0.83-1.28, P$ $=0.79$ ) in multivariable analysis (Fig. 4). In contrast, the 5-year and 10-year CMI of developing any hematologic SPM among aHSCT recipients was $1.3 \%(0.9-1.9 \%)$ and $2.7 \%(1.9-3.7 \%)$ compared to $0.8 \%(0.5-1.1 \%)$ and $1.1 \%$ (0.7-1.6\%) among non-aHSCT recipients (Fig. 2c; $P=$ 0.005 ) (Fig. 3c), corresponding to an aHR of 1.51 (1.01-2.27; $P=0.046)$ (Fig. 4).

\section{Discussion}

To our knowledge, this is the largest study examining the association between aHSCT and the development of SPMs in multiple myeloma patients. We observed that $6.6 \%$ of multiple myeloma patients developed a SPM 10 years after diagnosis and the risk of developing hematologic malignancies, but not solid tumors, was higher among those undergoing aHSCT. Specifically, we found an absolute increase of $1.3 \%$ in hematologic malignancies (from $0.8 \%$ among non-aHSCT recipients to $2.1 \%$ among aHSCT recipients). Despite this increased risk, myelomaspecific and non-cancer mortality greatly outweigh the risk of developing SPMs, and the 10 year mortality due to SPM was low, suggesting that benefits of aHSCT ${ }^{8-12,40}$ outweigh risks due to SPMs.

Two prior observational studies have addressed the risk of developing SPMs in multiple myeloma patients who have undergone aHSCT. In the United States, the Centers for International Blood \& Marrow Research (CIBMTR) database identified 4161 aHSCT recipients between 
Table 1 Baseline multiple myeloma characteristics by autologous stem cell transplant (aHSCT) among 1-year survivors of first primary multiple myeloma in California, 1991-2013.

\begin{tabular}{|c|c|c|c|c|c|c|c|}
\hline & \multirow{2}{*}{$\begin{array}{l}\text { All } \\
N\end{array}$} & \multirow{2}{*}{$\begin{array}{l}\text { aHSCT } \\
\%\end{array}$} & \multirow[b]{2}{*}{$N$} & \multirow[b]{2}{*}{ col \% } & \multicolumn{3}{|c|}{ No aHSCT } \\
\hline & & & & & $N$ & col \% & $P$-value \\
\hline All & 16,331 & 100.0 & 3202 & 100.0 & 13,129 & 100.0 & \\
\hline \multicolumn{8}{|l|}{ Gender } \\
\hline Male & 8767 & 53.7 & 1868 & 58.3 & 6899 & 52.5 & $<0.0001$ \\
\hline Female & 7564 & 46.3 & 1334 & 41.7 & 6230 & 47.5 & $<0.0001$ \\
\hline \multicolumn{8}{|l|}{ Race/Ethnicity } \\
\hline Non-Hispanic White & 9591 & 58.7 & 1901 & 59.4 & 7690 & 58.6 & 0.4117 \\
\hline African-American & 2115 & 13.0 & 358 & 11.2 & 1757 & 13.4 & 0.0009 \\
\hline Hispanic & 2954 & 18.1 & 649 & 20.3 & 2305 & 17.6 & 0.0004 \\
\hline Asian/Pacific Islander & 1467 & 9.0 & 283 & 8.8 & 1184 & 9.0 & 0.7495 \\
\hline Other/Unknown & 204 & 1.2 & 11 & 0.3 & 193 & 1.5 & $<0.0001$ \\
\hline \multicolumn{8}{|l|}{ Age diagnosis } \\
\hline Age $<40$ & 325 & 2.0 & 137 & 4.3 & 188 & 1.4 & $<0.0001$ \\
\hline $40-49$ & 1504 & 9.2 & 672 & 21.0 & 832 & 6.3 & $<0.0001$ \\
\hline $50-59$ & 3569 & 21.9 & 1322 & 41.3 & 2247 & 17.1 & $<0.0001$ \\
\hline $60-69$ & 4744 & 29.0 & 982 & 30.7 & 3762 & 28.7 & 0.0244 \\
\hline 70-79 & 4257 & 26.1 & 88 & 2.7 & 4169 & 31.8 & $<0.0001$ \\
\hline $80-89$ & 1815 & 11.1 & 1 & 0.0 & 1814 & 13.8 & $<0.0001$ \\
\hline$\geq 90$ & 117 & 0.7 & & & 117 & 0.9 & $<0.0001$ \\
\hline \multicolumn{8}{|c|}{ Treatment era of diagnosis } \\
\hline 1991-1997 & 3949 & 24.2 & 372 & 11.6 & 3577 & 27.2 & $<0.0001$ \\
\hline 1998-2002 & 3268 & 20.0 & 585 & 18.3 & 2683 & 20.4 & 0.006 \\
\hline $2003-2007$ & 3856 & 23.6 & 971 & 30.3 & 2885 & 22.0 & $<0.0001$ \\
\hline 2008-2013 & 5258 & 32.2 & 1274 & 39.8 & 3984 & 30.3 & $<0.0001$ \\
\hline \multicolumn{8}{|l|}{ 1st Course of treatment } \\
\hline \multicolumn{8}{|l|}{ Chemotherapy } \\
\hline Yes & 12,071 & 73.9 & 3007 & 93.9 & 9064 & 69.0 & $<0.0001$ \\
\hline No & 4023 & 24.6 & 187 & 5.8 & 3836 & 29.2 & $<0.0001$ \\
\hline Unknown & 237 & 1.5 & 8 & 0.2 & 229 & 1.7 & $<0.0001$ \\
\hline \multicolumn{8}{|l|}{ Radiation } \\
\hline Yes & 3,824 & 23.4 & 946 & 29.5 & 2878 & 21.9 & $<.0001$ \\
\hline No & 12,499 & 76.5 & 2256 & 70.5 & 10,243 & 78.0 & $<.0001$ \\
\hline Unknown & 8 & 0.0 & & & 8 & 0.1 & 0.1624 \\
\hline \multicolumn{8}{|c|}{ Neighborhood socioeconomic status (quintiles) } \\
\hline 1-Lowest & 2374 & 14.5 & 366 & 11.4 & 2008 & 15.3 & $<0.0001$ \\
\hline 2 & 2854 & 17.5 & 476 & 14.9 & 2378 & 18.1 & $<0.0001$ \\
\hline 3 & 3333 & 20.4 & 655 & 20.5 & 2678 & 20.4 & 0.9414 \\
\hline 4 & 3755 & 23.0 & 804 & 25.1 & 2951 & 22.5 & 0.0015 \\
\hline
\end{tabular}


Table 1 continued

\begin{tabular}{|c|c|c|c|c|c|c|c|}
\hline & \multirow{2}{*}{$\begin{array}{l}\text { All } \\
N\end{array}$} & \multirow{2}{*}{$\begin{array}{l}\text { aHSCT } \\
\%\end{array}$} & \multirow[b]{2}{*}{$N$} & \multirow[b]{2}{*}{ col \% } & \multicolumn{3}{|c|}{ No aHSCT } \\
\hline & & & & & $N$ & col \% & $P$-value \\
\hline 5-Highest & 4015 & 24.6 & 901 & 28.1 & 3114 & 23.7 & $<0.0001$ \\
\hline \multicolumn{8}{|l|}{ Insurance coverage } \\
\hline No insurance/Self pay & 190 & 1.2 & 22 & 0.7 & 168 & 1.3 & 0.0051 \\
\hline Private insurance & 7256 & 44.4 & 2154 & 67.3 & 5102 & 38.9 & $<0.0001$ \\
\hline Medicaid/Gov & 1255 & 7.7 & 314 & 9.8 & 941 & 7.2 & $<0.0001$ \\
\hline Medicare & 5570 & 34.1 & 586 & 18.3 & 4984 & 38.0 & $<0.0001$ \\
\hline Unknown insurance & 2059 & 12.6 & 126 & 3.9 & 1933 & 14.7 & $<0.0001$ \\
\hline \multicolumn{8}{|l|}{ Comorbidities $^{a}$} \\
\hline No admissions & 7445 & 45.6 & 1613 & 50.4 & 5832 & 44.4 & $<0.0001$ \\
\hline 0 Comorbidities & 1511 & 9.3 & 412 & 12.9 & 1099 & 8.4 & $<0.0001$ \\
\hline 1-2 Comorbidities & 3614 & 22.1 & 700 & 21.9 & 2914 & 22.2 & 0.6833 \\
\hline$\geq 3$ Comorbidities & 3761 & 23.0 & 477 & 14.9 & 3284 & 25.0 & $<0.0001$ \\
\hline \multicolumn{8}{|l|}{ SPM site } \\
\hline All & 933 & 5.7 & 186 & 5.8 & 747 & 5.7 & 0.7945 \\
\hline Digestive system & 184 & 1.1 & 22 & 0.7 & 162 & 1.2 & 0.0025 \\
\hline Breast & 97 & 0.6 & 18 & 0.6 & 79 & 0.6 & 0.7195 \\
\hline Respiratory system & 119 & 0.7 & 19 & 0.6 & 100 & 0.8 & 0.2459 \\
\hline Leukemia & 81 & 0.5 & 32 & 1.0 & 49 & 0.4 & $<0.0001$ \\
\hline Lymphoma & 40 & 0.2 & 6 & 0.2 & 34 & 0.3 & 0.4245 \\
\hline MDS & 31 & 0.2 & 8 & 0.2 & 23 & 0.2 & 0.4054 \\
\hline Female genitals & 36 & 0.2 & 9 & 0.3 & 27 & 0.2 & 0.4379 \\
\hline Urinary system & 66 & 0.4 & 7 & 0.2 & 59 & 0.4 & 0.0491 \\
\hline Male genitals & 115 & 0.7 & 31 & 1.0 & 84 & 0.6 & 0.0442 \\
\hline Brain and other CNS & 7 & 0.0 & - & - & 7 & 0.1 & 0.1851 \\
\hline Endocrine system & 19 & 0.1 & 6 & 0.2 & 13 & 0.1 & 0.1993 \\
\hline Soft tissue & 4 & 0.0 & 2 & 0.1 & 2 & 0.0 & 0.1315 \\
\hline Bone and joint & 1 & 0.0 & - & - & 1 & 0.0 & 0.6176 \\
\hline Oral cavity system & 29 & 0.2 & 9 & 0.3 & 20 & 0.2 & 0.1286 \\
\hline Skin & 75 & 0.5 & 15 & 0.5 & 60 & 0.5 & 0.9884 \\
\hline Mesothelioma & 4 & 0.0 & 1 & 0.0 & 3 & 0.0 & 0.7994 \\
\hline Miscellaneous & 25 & 0.2 & 1 & 0.0 & 24 & 0.2 & 0.0432 \\
\hline
\end{tabular}

nSES neighborhood socioeconomic status, SPM second primary malignancy, MDS myelodysplastic syndrome.

${ }^{a}$ Comorbidities were calculated within 2 years prior to multiple myeloma diagnosis.

1990-2010. After accounting for the competing risk of death, 2.6 and $5.1 \%$ developed a SPM 3 and 7 years postaHSCT. When compared to the rates of acute myeloid leukemia and myelodysplastic syndrome development in the general population, significant increased risk was identified (standardized incidence ratios 5.19 and 85.5, respectively) ${ }^{20}$. While our findings were concordant, it is notable that the differential risk of developing a hematologic SPM was much lower in our study, likely due to our comparison group of multiple myeloma patients having a higher risk of developing these diseases when compared to the general population. A sub-study of the European Bone Marrow Transplant Registry identified 3204 multiple myeloma patients, of whom 135 developed SPMs. At 6 


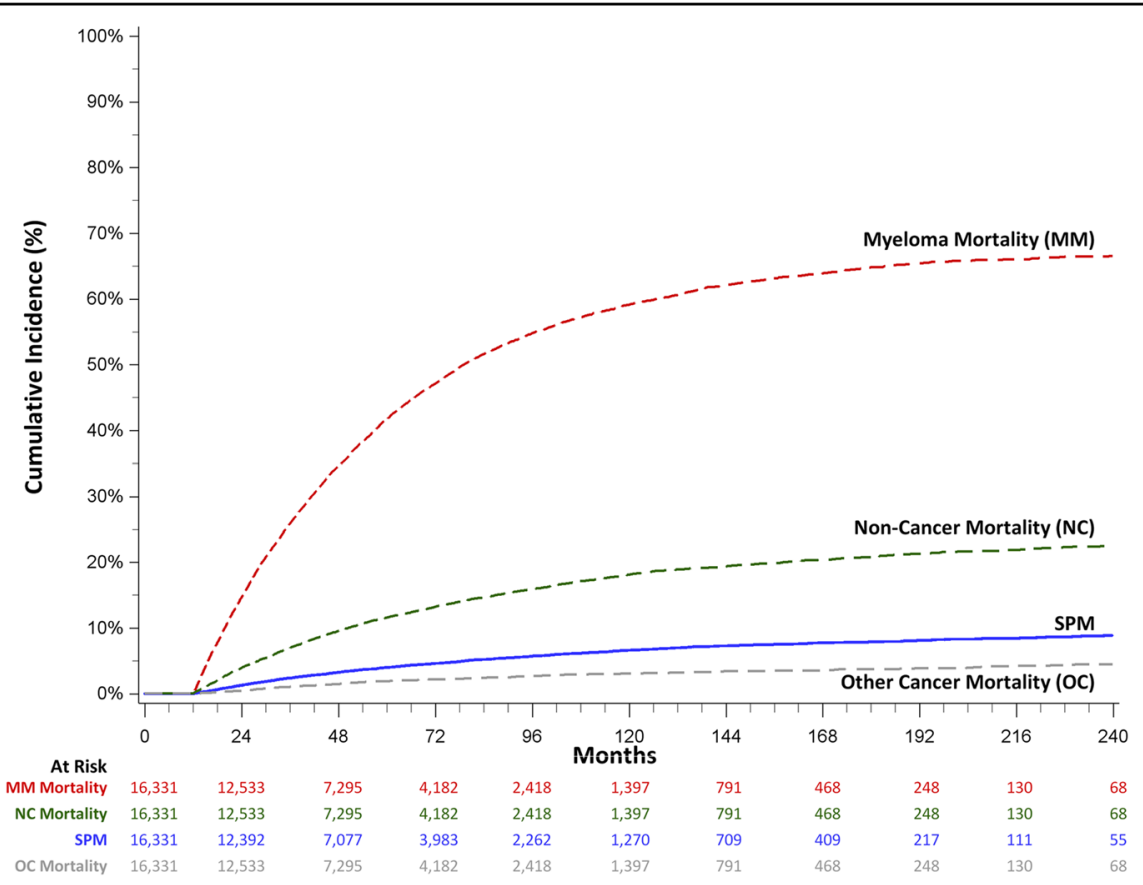

Fig. 1 Cumulative incidence of mortality and second primary malignancy (SPM) among 1-year survivors of first primary multiple myeloma in California, 1991-2013. The cumulative incidence of second primary malignancy (SPM) development accounting for the competing risk of death from multiple myeloma (MM), non-cancer related mortality (NC) and other cancer mortality (OC).

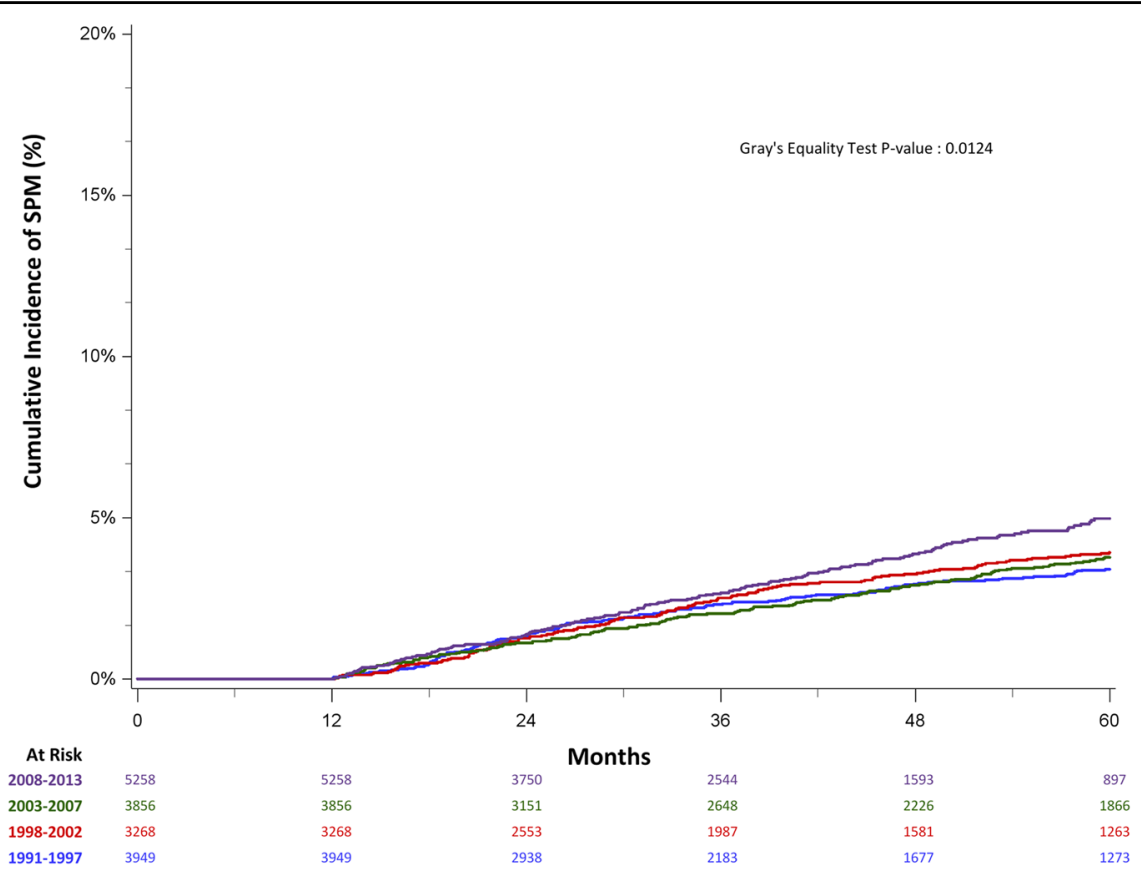

Fig. 2 Five-year cumulative incidence of second primary malignancy by treatment era among 1-year survivors of first primary multiple myeloma in California, 1991-2013. The cumulative incidence of developing second primary malignancies in patient cohorts diagnosed between 1991-1997, 1998-2002, 2003-2007, 2008-2013, accounting for the competing risk of death. The 5 year cumulative incidence of SPM development was higher in those diagnosed between 2008-2013 [5.0\% (4.3-5.8\%)] compared to earlier eras: 3.8\% (3.2-4.4\%), 3.9\% (3.3-4.6\%), 3.4\% (2.9-4.0\%) in 2003-2007, 1998-2002, and 1991-1997. 

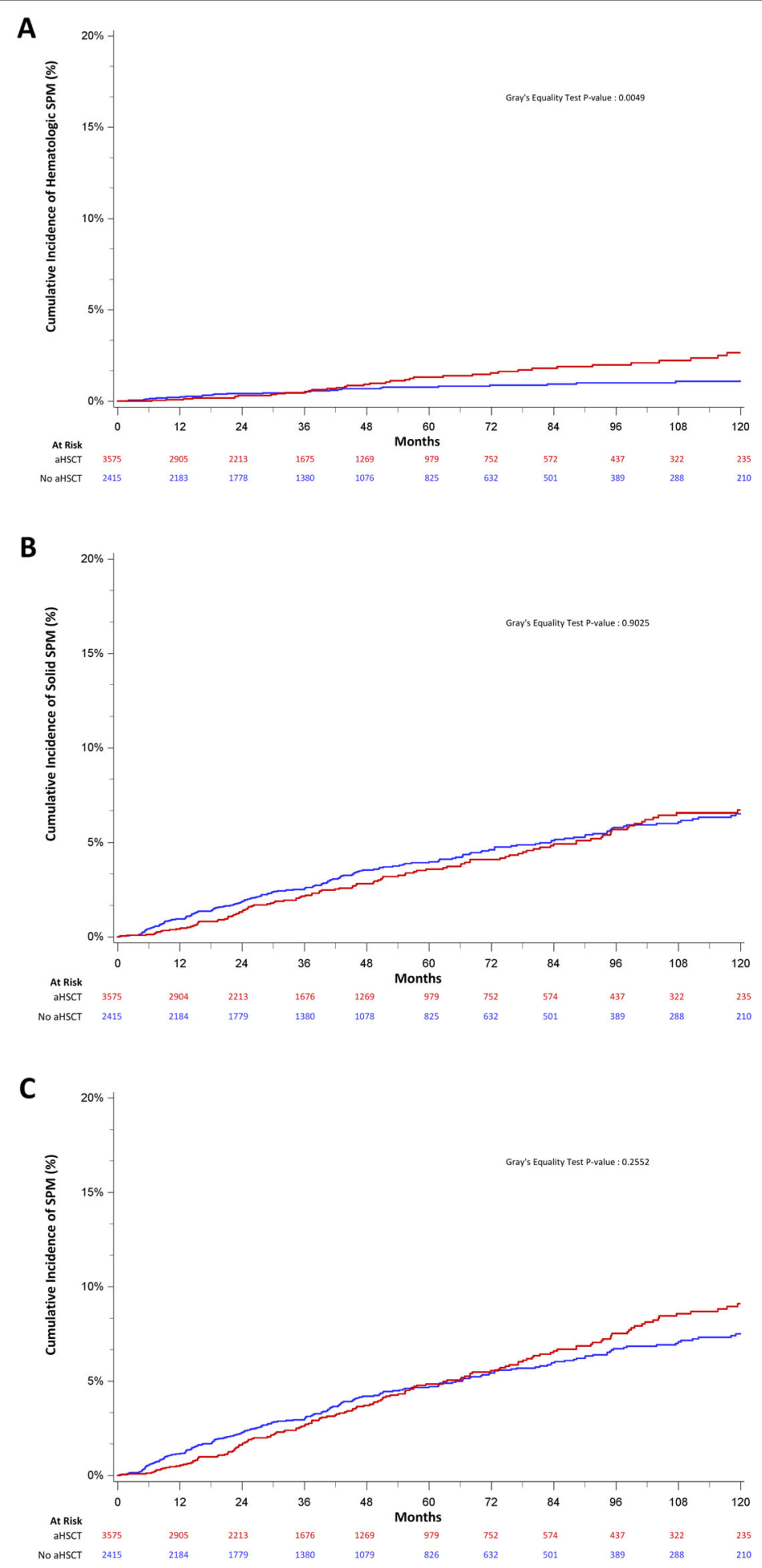

Fig. 3 Cumulative incidence of second primary malignancy development among multiple myeloma patients with or without autologous stem cell transplant. Overall solid hematologic footnote: A overall second primary malignancy (SPM), B Solid tumor SPM, and C Hematologic SPM by autologous stem cell transplant (aHSCT) treatment among matched 1-year survivors with first primary multiple myeloma in California, 1991-2013. aHSCT patients were matched to two patients without aHSCT on age, year of diagnosis, race/ethnicity, nSES status comorbidities, and follow-up time. Person time was calculated from aHSCT date to SPM, death, or last known follow-up from the cancer registry. 
aHSCT Treatment vs. No aHSCT Treatment

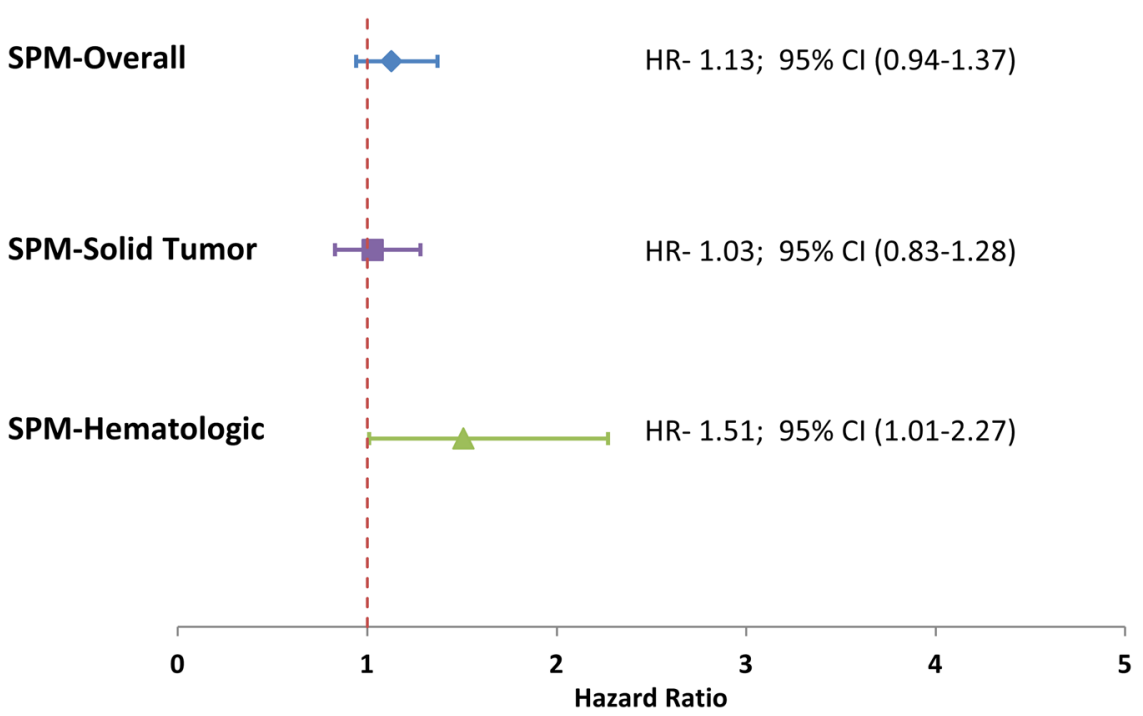

Fig. 4 Adjusted effect of autologous stem cell transplant (aHSCT) treatment compared to no aHSCT treatment on the risk of second primary malignancy (SPM) among 1-year survivors with first primary multiple myeloma in California, 1991-2014. aHSCT was included as a time dependent covariate. Adjusted for the competing risk of death and baseline multiple myeloma characteristics (gender, race/ethnicity, age at diagnosis, treatment era of diagnosis, initial course of treatment, neighborhood socioeconomic status, comorbidities, initial health insurance, marital status, and urban vs. rural location of residence).

years, the cumulative incidence, accounting for the competing risk of death, was $5.3 \%$ overall (1.4\% for hematologic malignancies and $3.6 \%$ for solid tumors). The current study found similar 5 -year overall cumulative incidence rate of $4.0 \%$ (0.6\% hematologic and $3.4 \%$ solid tumors).

Multiple prior population-based studies have examined the relationship between multiple myeloma and SPM development. An analysis of the SEER program between 1973-2008 showed that myeloma patients had a nearly $10 \%$ lower incidence of solid tumor development than the background population. In distinction, they found a more than a $60 \%$ increased incidence of developing hematologic malignancies, both myeloid and lymphoid: SIR 1.63 (1.45-1.84), similar to our findings. When SPM rates were compared across treatment eras, no change in the risk of SPM development was identified ${ }^{21,23}$. An analysis of the Swedish healthcare registry and a registry of MGUS patients diagnosed between 1986-2005 reported a nearly $30 \%$ increased risk of SPMs in multiple myeloma patients (SIR 1.26 [1.16-1.36]). This effect was particularly pronounced for hematologic malignancies, with a more than 2-fold increased incidence (SIR 2.04 [1.59-2.58]), and no differences in incidence was observed over time. Interestingly, MGUS patients had an increased incidence of AML, MDS, and myeloproliferative neoplasms (MPNs), implying that an underlying bone marrow process or stem cell defect may link these diagnoses, rather than treatment $^{26}$. An analysis comparing SPM rates for MM patients in German and Swedish national cancer registries found different distributions of SPMs in the two populations-though an increased incidence of AML was observed in both ${ }^{18}$. Thus, factors that have yet to be elucidated are also likely involved in SPM development in multiple myeloma patients.

We found that the rates of SPM development have increased in the most recent era. However, this finding needs to be considered preliminary and hypothesis generating until more complete follow-up allows for further study. Differences in the findings of the current and prior studies may be due to differences in analytic methods, background population risk of SPM development, followup time, or latency periods, along with the inclusion of a more modern study population with different drug exposures, such as incorporation of post-aHSCT maintenance, and trends in cancer screening along with an increasing awareness of SPM risk in multiple myeloma patients. Future studies focusing on changing trends in SPM development are needed to identify populations at highest risk to better inform both patients and physicians, and allow for targeted screening of multiple myeloma patients.

RCTs of continuous lenalidomide therapy have observed higher SPM rates among patients undergoing lenalidomide maintenance after aHSCT, but not in transplant ineligible patients undergoing continuous lenalidomide based therapy compared to placebo ${ }^{31-33,41,42}$. A metaanalysis of lenalidomide trials identified patients receiving 
oral melphalan and lenalidomide at highest risk for developing SPMs, though those receiving intravenous melphalan and lenalidomide had numerically higher incidence of SPM development than those not receiving lenalidomide ${ }^{22}$. The current study found lower rates of SPM development after aHSCT than was seen in RCTs. This may be related to several factors, including higher competing risks of death in the general population compared to populations included in $\mathrm{RCTs}^{43}$, variable adoption of maintenance therapy in the general population, potentially shorter duration of maintenance therapy, and our use of a conservative exclusion of SPMs diagnosed within 12 months of multiple myeloma diagnosis. The current study endeavored to estimate the contribution of high dose chemotherapy to SPM development, and thus addresses a different question than recent RCTs, and, lacking additional treatment data, we cannot assess the contribution of post-aHSCT lenalidomide maintenance to SPM development.

The current study has several limitations. All observational studies of treatment modalities are subject to selection bias. However, it is unlikely that physicians accounted for risk of SPM when offering aHSCT to patients, as relative contribution of aHSCT to the risk of SPM development is poorly defined. Second, the combined CCR-OSHPD data base does not include detailed treatment information, so we cannot account for various induction and maintenance strategies. The chemotherapy variable is applicable only to first course therapy, not later lines of therapy, and does not have additional details available. As lenalidomide maintenance after aHSCT, and the combination of low dose melphalan and lenalidomide, have been associated with SPM development, there is a potential for unmeasured confounding if patients undergoing aHSCT were more likely to receive lenalidomide maintenance $22,28,29,32$. However, practice patterns have indicated a rapid adoption of novel agents in the treatment of multiple myeloma, arguing that most patients would have had at least some exposure during the modern treatment eras to immunomodulatory agents ${ }^{44,45}$. Common use of post-aHSCT lenalidomide maintenance starting in 2012 may have resulted in increased SPM rates in the most recent time frame. The current data does not include detailed treatments, so this hypothesis cannot be directly tested, but deserves future analysis with more mature follow up and treatment data. While patients undergoing aHSCT may live longer, we addressed this potential source of survival time bias by accounting for the competing risk of death, treating aHSCT as a time dependent covariate in regression analyses, and excluding patients who died within a year of diagnosis. We also undertook a conservative approach to exclude SPMs diagnosed within 1 year of MM diagnosis to ensure that we did not count concurrent primaries, or asymptomatic malignancies identified during MM work-up or during initial treatment periods when oncologic follow-up is likely to be frequent. The CCR relies on death certificate data to assign a cause of death. This may be a source of attribution bias when considering cause-specific mortality. The current study also has several strengths. By including all multiple myeloma cases diagnosed in California, we have been able to compare rates of SPM development among myeloma patients undergoing a specific treatment with relatively long follow-up times, rather than comparing to the general population. To our knowledge using multiple myeloma patients as comparators, rather than the background population, to assess the association between aHSCT and SPM development is unique in the literature, and likely provides an estimation of the risk associated with aHSCT in these patients. California is one of the most populous and diverse states in the United States, likely increasing generalizability.

In conclusion, our study agrees with prior studies which have identified an increased risk of hematologic SPMs in multiple myeloma patients. The increased risk among aHSCT recipients compared to non-aHSCT recipients is an important consideration for patients undergoing the procedure. However, while the increased risk of hematologic SPMs is high in relative terms, the absolute risk increase over the subsequent 10 years is small, and, in our opinion, outweighed by the potential benefits to both overall and progression-free survival conferred by aHSCT use $^{8-12}$. As MM patients continue to live longer, robust data on SPM risks associated with various treatment strategies are necessary for high quality, informed shared decision making.

\section{Acknowledgements}

This work was supported in part by the UC Davis Paul Calabresi Career Development Award for Clinical Oncology as funded by the National Cancer Institute/National Institutes of Health through grant \#5K12-CA138464 (A.S.R.). The collection of cancer incidence data used in this study was supported by the California Department of Public Health pursuant to California Health and Safety Code Section 103885; Centers for Disease Control and Prevention's (CDC) National Program of Cancer Registries, under cooperative agreement 5NU58DP006344; the National Cancer Institute's Surveillance, Epidemiology and End Results Program under contract HHSN261201800032l awarded to the University of California, San Francisco, contract HHSN261201800015I awarded to the University of Southern California, and contract HHSN261201800009l awarded to the Public Health Institute. The ideas and opinions expressed herein are those of the author(s) and do not necessarily reflect the opinions of the State of California, Department of Public Health, the National Cancer Institute, and the Centers for Disease Control and Prevention or their Contractors and Subcontractors. UL1 00001860 National Center for Advancing Translational Research (NCATS), National Institutes of Health (TW). A.S.R.: Consultant/Advisor: Amgen, Seattle Genetics; research funding: Amgen, Speakers Bureau: Millennium/Takeda, Janssen B.A.J.: Served as consultant/ advisor for AbbVie, Amgen, Celgene, GlycoMimetics, Jazz, Pharmacyclics, Tolero and Treadwell; received travel support from AbbVie, Amgen, and Glycomimetics; and received research funding to his institution from AbbVie, Accelerated Medical Diagnostics, AROG, Celgene, Daiichi Sankyo, F. Hoffmann- 
La Roche, Forma, Genentech/Roche, GlycoMimetics, Hanmi, Incyte, Jazz, LP Therapeutics, Pfizer, Pharmacyclics and Sigma Tau. T.W.: Steering committees: Pfizer, Janssen

\section{Author details}

${ }^{1}$ Davis School of Medicine, Center for Hematology and Oncology Outcomes Research and Training (COHORT), University of California, Sacramento, CA, USA ${ }^{2}$ Davis Comprehensive Cancer Center, University of California, Sacramento, CA, USA. ${ }^{3}$ Northern California Department of Veterans Affairs, Sacramento, CA, USA

\section{Conflict of interest}

The authors declare that they have no conflict of interest.

\section{Publisher's note}

Springer Nature remains neutral with regard to jurisdictional claims in published maps and institutional affiliations.

Supplementary Information accompanies this paper at (https://doi.org/ 10.1038/s41408-020-00400-4).

Received: 24 July 2020 Revised: 16 November 2020 Accepted: 7 December 2020

Published online: 07 January 2021

\section{References}

1. Costa, L. J. et al. Recent trends in multiple myeloma incidence and survival by age, race, and ethnicity in the United States. Blood Adv. 1, 282-287 (2017).

2. Kumar, S. K. et al. Improved survival in multiple myeloma and the impact of novel therapies. Blood 111, 2516-2520 (2008).

3. Kumar, S. K. et al. Continued improvement in survival in multiple myeloma: changes in early mortality and outcomes in older patients. Leukemia $\mathbf{2 8}$ 1122-1128 (2014)

4. Dimopoulos, M. A., Richardson, P. G., Moreau, P. \& Anderson, K. C. Current treatment landscape for relapsed and/or refractory multiple myeloma. Nat. Rev. Clin. Oncol. 12, 42-54 (2015).

5. Lokhorst, H. M. et al. Targeting CD38 with daratumumab monotherapy in multiple myeloma. N. Engl. J. Med. 373, 1207-1219 (2015).

6. Lonial, S. et al. Elotuzumab therapy for relapsed or refractory multiple myeloma. N. Engl. J. Med. 373, 621-631 (2015).

7. Attal, M. et al. A prospective, randomized trial of autologous bone marrow transplantation and chemotherapy in multiple myeloma. Intergroupe Francais du Myelome. N. Engl. J. Med. 335, 91-97 (1996).

8. Palumbo, A. et al. Autologous transplantation and maintenance therapy in multiple myeloma. N. Engl. J. Med. 371, 895-905 (2014).

9. Gay, F. et al. Chemotherapy plus lenalidomide versus autologous transplantation, followed by lenalidomide plus prednisone versus lenalidomide maintenance, in patients with multiple myeloma: a randomised, multicentre, phase 3 trial. Lancet Oncol. 16, 1617-1629 (2015).

10. Attal, M. et al. Lenalidomide, bortezomib, and dexamethasone with transplantation for myeloma. N. Engl. J. Med. 376, 1311-1320 (2017).

11. Winn, A. N., Shah, G. L., Cohen, J. T., Lin, P. J. \& Parsons, S. K. The real world effectiveness of hematopoietic transplant among elderly individuals with multiple myeloma. J. Natl Cancer Inst. https://doi.org/10.1093/jnci/djv139 (2015).

12. Rosenberg, A. S., Brunson, A., Jonas, B. A., Keegan, T. H. M. \& Wun, T. Association between autologous stem cell transplant and survival among Californians with multiple myeloma. J. Natl Cancer Inst. 111, 78-85 (2019).

13. Bilotti, E. et al. Sunvivorship care guidelines for patients living with multiple myeloma: consensus statements of the International Myeloma Foundation Nurse Leadership Board. Clin. J. Oncol. Nurs. 15, 5-8 (2011).

14. Bilotti, E., Gleason, C. L. \& McNeill, A. International Myeloma Foundation Nurse Leadership B. Routine health maintenance in patients living with multiple myeloma: survivorship care plan of the International Myeloma Foundation Nurse Leadership Board. Clin. J. Oncol. Nurs. 15, 25-40 (2011).

15. Sahebi, F. et al. Incidence of second primary malignancies after autologous transplantation for multiple myeloma in the era of novel agents. Biol. Blood Marrow Transpl. 24, 930-936 (2018).
16. Musto, P. et al. Second primary malignancies in multiple myeloma: an overview and IMWG consensus. Ann. Oncol. 28, 228-245 (2017).

17. Castillo, J. J. \& Gertz, M. A. Secondary malignancies in patients with multiple myeloma, Waldenstrom macroglobulinemia and monoclonal gammopathy of undetermined significance. Leuk. Lymphoma 58, 773-780 (2017).

18. Chen, T. et al. Risk of second primary cancers in multiple myeloma survivors in German and Swedish Cancer Registries. Sci. Rep. 6, 22084 (2016).

19. Areethamsirikul, N. \& Reece, D. E. The risk of secondary primary malignancies after therapy for multiple myeloma. Leuk. Lymphoma 56, 3012-3021 (2015).

20. Mahindra, A. et al. New cancers after autotransplantations for multiple myeloma. Biol. Blood Marrow Transpl. 21, 738-745 (2015).

21. Ailawadhi, S., Swaika, A., Razavi, P., Yang, D. \& Chanan-Khan, A. Variable risk of second primary malignancy in multiple myeloma patients of different ethnic subgroups. Blood Cancer J. 4, e243 (2014).

22. Palumbo, A. et al. Second primary malignancies with lenalidomide therapy for newly diagnosed myeloma: a meta-analysis of individual patient data. Lancet Oncol. 15, 333-342 (2014).

23. Razavi, P. et al. Patterns of second primary malignancy risk in multiple myeloma patients before and after the introduction of novel therapeutics. Blood Cancer J. 3, e121 (2013).

24. Chakraborty, S., Hauke, R. J., Bonthu, N. \& Tarantolo, S. R. Increased incidence of a second lymphoproliferative malignancy in patients with multiple myeloma-a SEER based study. Anticancer Res 32, 4507-4515 (2012).

25. Tzeng, H. E. et al. Time trend of multiple myeloma and associated secondary primary malignancies in Asian patients: a Taiwan population-based study. PLoS ONE 8, e68041 (2013).

26. Mailankody, S. et al. Risk of acute myeloid leukemia and myelodysplastic syndromes after multiple myeloma and its precursor disease (MGUS). Blood 118, 4086-4092 (2011).

27. Poh, C., Keegan, T. \& Rosenberg, A. S. Second primary malignancies in multiple myeloma: a review. Blood Rev. https://doi.org/10.1016/j.blre.2020.100757 (2020).

28. Dimopoulos, M. A. et al. A review of second primary malignancy in patients with relapsed or refractory multiple myeloma treated with lenalidomide. Blood 119, 2764-2767 (2012).

29. McCarthy, P. L. et al. Lenalidomide maintenance after autologous stem-cell transplantation in newly diagnosed multiple myeloma: a meta-analysis. J. Clin. Oncol. 35, 3279-3289 (2017).

30. Govindarajan, R. et al. Preceding standard therapy is the likely cause of MDS after autotransplants for multiple myeloma. Br. J. Haematol. 95, 349-353 (1996).

31. Attal, M. et al. Lenalidomide maintenance after stem-cell transplantation for multiple myeloma. N. Engl. J. Med. 366, 1782-1791 (2012).

32. Holstein, S. A. et al. Updated analysis of CALGB (Alliance) 100104 assessing lenalidomide versus placebo maintenance after single autologous stem-cell transplantation for multiple myeloma: a randomised, double-blind, phase 3 trial. Lancet Haematol 4, e431-e442 (2017).

33. McCarthy, P. L. et al. Lenalidomide after stem-cell transplantation for multiple myeloma. N. Engl. J. Med. 366, 1770-1781 (2012).

34. Costa, L. J. et al. Second primary malignancy after multiple myelomapopulation trends and cause-specific mortality. Br. J. Haematol. 182, 513-520 (2018).

35. Tao, L., Foran, J. M., Clarke, C. A., Gomez, S. L. \& Keegan, T. H. Socioeconomic disparities in mortality after diffuse large B-cell lymphoma in the modern treatment era. Blood 123, 3553-3562 (2014).

36. Yost, K., Perkins, C., Cohen, R., Morris, C. \& Wright, W. Socioeconomic status and breast cancer incidence in California for different race/ethnic groups. Cancer Causes Control 12, 703-711 (2001).

37. Surveillance E., and End Results Program. Site Recode ICD-O-3/WHO 2008 Definition. In, 2008. https://seer.cancer.gov/siterecode/icdo3_dwhoheme/ index.html.

38. Schoenman, J. A., Sutton, J. P., Elixhauser, A. \& Love, D. Understanding and enhancing the value of hospital discharge data. Med. Care Res. Rev. 64, 449-468 (2007)

39. Allison, P. D. Survival Analysis Using SAS: A Practical Guide 2nd edn (SAS Institute, Cary, 2010).

40. Dhakal, B. et al. Autologous transplantation for newly diagnosed multiple myeloma in the era of novel agent induction: a systematic review and metaanalysis. JAMA Oncol. 4, 343-350 (2018).

41. Palumbo, A. et al. Continuous lenalidomide treatment for newly diagnosed multiple myeloma. N. Engl. J. Med. 366, 1759-1769 (2012). 
42. Benboubker, L. et al. Lenalidomide and dexamethasone in transplant-ineligible patients with myeloma. N. Engl. J. Med. 371, 906-917 (2014).

43. Costa, L. J., Hari, P. N. \& Kumar, S. K. Differences between unselected patients and participants in multiple myeloma clinical trials in US: a threat to external validity. Leuk. Lymphoma 57, 2827-2832 (2016).
44. Warren, J. L., Harlan, L. C., Stevens, J., Little, R. F. \& Abel, G. A. Multiple myeloma treatment transformed: a population-based study of changes in initial management approaches in the United States. J. Clin. Oncol. 31, 1984-1989 (2013).

45. Jagannath, $\mathrm{S}$. et al. Real-world treatment patterns and associated progressionfree survival in relapsed/refractory multiple myeloma among US community oncology practices. Expert Rev. Hematol. 9, 707-717 (2016). 